\title{
Review Article \\ “mTOR Signaling Pathway": A Potential Target of Curcumin in the Treatment of Spinal Cord Injury
}

\author{
Jingquan Lin, ${ }^{1}$ Xue Huo, ${ }^{2}$ and Xuehong Liu ${ }^{1}$ \\ ${ }^{1}$ Department of Histology and Embryology, Medical College, Shaoxing University, Zhejiang, China \\ ${ }^{2}$ Institute of Neuroscience, Zhejiang University School of Medicine, Hangzhou, China \\ Correspondence should be addressed to Xuehong Liu; liuxueh6588@126.com
}

Received 5 December 2016; Revised 15 April 2017; Accepted 18 May 2017; Published 12 June 2017

Academic Editor: Ayhan Cömert

Copyright (c) 2017 Jingquan Lin et al. This is an open access article distributed under the Creative Commons Attribution License, which permits unrestricted use, distribution, and reproduction in any medium, provided the original work is properly cited.

\begin{abstract}
The purpose of this review is to discuss the possibility of the treatment of spinal cord injury (SCI) with curcumin via regulating the MTOR signaling pathway, which may provide another strong support for curcumin to be a promising medicine applied to the treatment of SCI. Curcumin is termed as a multifunctional targeting therapy drug that regulates the mTOR signaling pathway in the treatment of numerous diseases. Previous research has already revealed that mTOR signaling pathway plays a vital role in prognosis, which involves the axon regeneration and autophagy. This review discusses a potential mechanism that curcumin suppresses the activation of this pathway and ameliorates the microenvironment of axons regeneration which would provide a new way that induces autophagy appropriately.
\end{abstract}

\section{Introduction}

Spinal cord injury (SCI) is a devastating central nervous system trauma, which impacts one's health both physically and mentally which caused the family and society to sink into a heavy burden, yet global prevalence and incidence of traumatic spinal cord injury indicates that millions of people have been suffering from SCI in past decades [1]. There are two pathological processes that appear when the spinal cord injury is acute damage; one is called "primary injury," which is caused by a sharp external force in constriction or laceration; this change is irreversible; the other also was deemed as the most terrible/serious one is "secondary injury," which is based on the "primary injury"; it results from intricate pathophysiological changes involving vascular dysfunction, inflammation, oxidative stress, excitotoxicity, apoptosis, and so on [2]. Because the "primary injury" is irreversible, the clinical treatments always paid more attention to the secondary injury to inhibit the pathophysiological change and attenuate the secondary injury of post-SCI so as to realize the function of neuroprotection and neuroregeneration. The mammalian target of rapamycin (mTOR) signaling pathway, which influenced cellular functions and metabolism, growth, proliferation, and viability, plays a crucial role in the functional recovery of central nervous system trauma, especially for axon regeneration and autophagy [3-5], which has an extensive link with apoptosis [6-8]. Thus, it will be a potential therapeutic target in the treatment of SCI. Curcumin (Diferuloylmethane, $\mathrm{C}_{21} \mathrm{H}_{20} \mathrm{O}_{6}$ ), a natural polyphenol extract from the plant Curcuma along with little side-effect as well as being multitarget, was reported to alleviate the secondary injury of post-SCI, which help the rehabilitation of neuron damage after SCI [9] (Figure 1). Moreover, Curcuma is a regulator controlling the mTOR signaling pathway in the treatments of diseases [10]. However, few researches relate to curcumin on mTOR signaling pathway after SCI has been reported. In this review, the related mechanism will be discussed.

\section{Pathophysiology of SCI}

The complex pathophysiology of SCI can be described as two stages: primary phase and secondary phase. Nevertheless, most clinical instances show that the secondary stage followed by the primary insult is more significant because the prognosis of SCI is mainly influenced by the secondary injury. Besides, it provides a vital information that might be a 


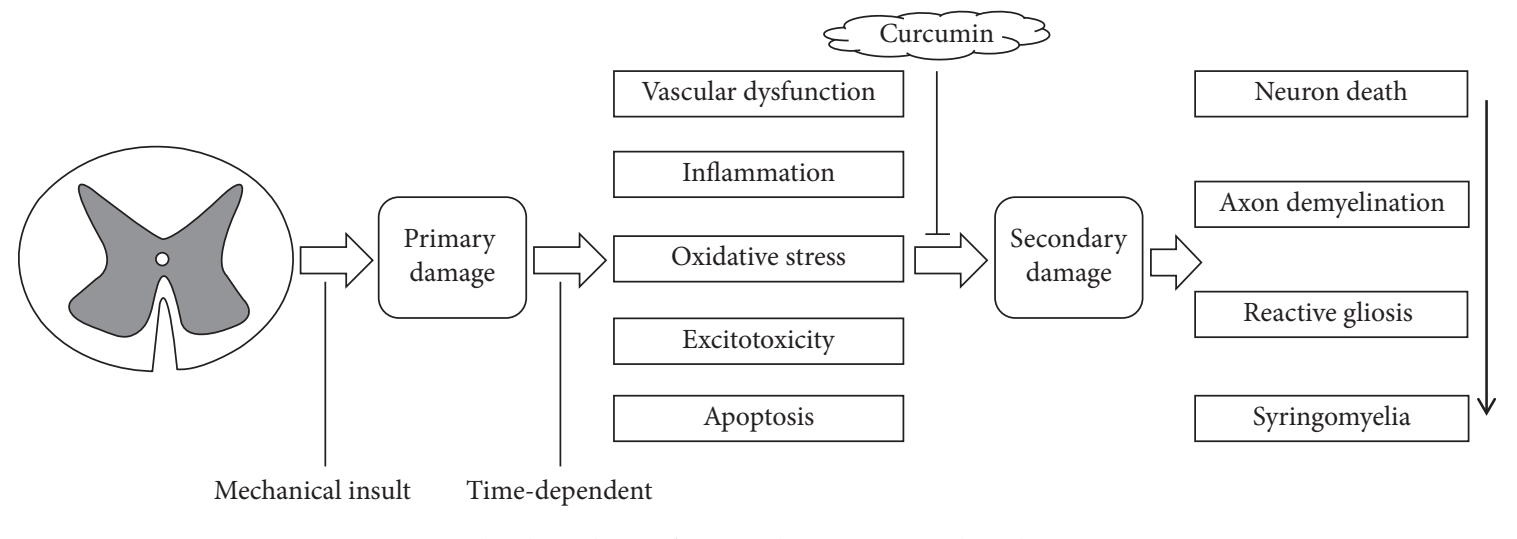

FIGURE 1: Pathophysiology of SCI and curcumin-induced action in SCI.

promising therapy for preventing deterioration of initial area of injury.

The secondary phase is a delayed and progressive tissue damage following the initial onset of SCI, which occurs over the time course of hours to weeks and is caused by a cascade of biological events. Following the mechanical insult, there is an unavoidable structural destroy and vascular dysfunction that end up with edema, necrosis and ischemia. Vascular dysfunction includes hemorrhage, vasospasm, thrombosis, loss of autoregulation, and disruption of blood spinal cord barrier. These impairments lead to more inflammatory cells across the defective barrier and expansion of the initial damage. The inflammatory response is regarded as an important contribution to secondary damage; inflammatory cells such as macrophages, microglia, $\mathrm{T}$ cells, and neutrophils infiltrate the lesion area, which is releasing inflammatory factors such as tumor necrosis factor- $\alpha$ (TNF- $\alpha$ ), interleukin(IL-) $1 \alpha$, IL- $1 \beta$, and IL- 6 , and lead to the disorder of the microenvironment of the spinal cord. Besides, infiltrating macrophages, neutrophils, and activated microglia also join the oxidative stress with providing extra sources of free radicals. Free radicals react with the polyunsaturated fatty acid of the cellular membrane yielding to the peroxidation and disorder of the normal phospholipid structure of cellular and subcellular organelle membranes, which significantly contributes to neural tissue damage. In particular, extracellular amino acids, glutamate and aspartate, are increasingly released from disorder cells. The excessive activation of relevant amino acid receptors produces excitotoxicity and leads to the loss of neurons both in necrosis and apoptosis. Apoptosis occurs in neurons, oligodendrocytes, microglia, and astrocytes. Oligodendrocytes are easily subjected to apoptotic loss that contributes to postinjury demyelination $[2,11]$.

Eventually, all of these processes bring neuron death, axon demyelination, reactive gliosis, and syringomyelia around the lesion. Considering the pathophysiology of SCI, alleviating secondary damage is a key point to the treatment (Figure 1).

\section{3. mTOR Signaling Pathway and SCI}

mTOR signaling pathway is a promising pharmacological option that is well known for its roles in the process of growing cells, metabolism, and cancer. mTOR, is a core of the signaling networks, which belongs to the phosphoinositide 3-kinase- (PI3K-) related protein kinase (PIKK) family. It assembles into two complexes, mTORC1 and mTORC2, which were composed of its subunit raptor and rictor. Respectively, both contained mLST8 subunit, with distinct inputs and downstream effects [12-15]. Likewise, mTOR has an instrumental role in a great deal of physiological functions of the central nervous system, including the regulation of neuronal cell growth, survival, axonal and dendritic development during differentiation, and synaptic plasticity [16, 17]. Significantly, this pathway is earning novel concern for its role in the traumatic CNS injury repair and regeneration like traumatic brain injury (TBI) and SCI [3-5].

3.1. Promoting Axon Regeneration Dependent on Inhibiting Glial Scar Formation via Inhibiting the Pathway after SCI. Glial scar, an extrinsic environment for axon regeneration, acts like physical and chemical barriers and is mainly composed of reactive astrocytes, with the upregulation of glial fibrillary acidic protein (GFAP) and vimentin, the hallmarks of the gliosis process, and extracellular matrix molecules particularly interact with the chondroitin sulfate proteoglycans (CSPGs), which is degenerated by Chondroitinase ABC $(\mathrm{ChABC})$ allowing axon regeneration and function recovery in SCI [18]. Nevertheless, some findings indicated the diminished spread of the damage by limiting the areas of spinal cord injury, thereby suppressing the axons regeneration and function recovery. MTOR-STAT3 pathway is activated while Nogo receptors have been blocked by Nogo-66. MTORSTAT3 pathway promotes neural progenitors that differentiate into lineages of astrocytes [19]. The activation of the $\mathrm{PI} 3 \mathrm{~K} / \mathrm{Akt} / \mathrm{mTOR}$ signaling pathway involves the formation of glial scar. Rapamycin, as an mTORC1 sensitive allosteric inhibitor, combined with the intracellular $12 \mathrm{kDa}$ FK506binding protein (FKBP12), suppressed the proliferation of astrocyte and reduced the GFAP positive cells at the damaged area that followed with the progress of axon's reconstruction [20]. Similarly, stimulating the expression of phosphatase and tensin homolog deleted on chromosome 10 (PTEN) inhibits the PI3K/Akt/mTOR signaling pathway and attenuates the formation of glial scar, which may be activated in astrocytes in 3 days after SCI involving gliosis, when decreasing GFAP 


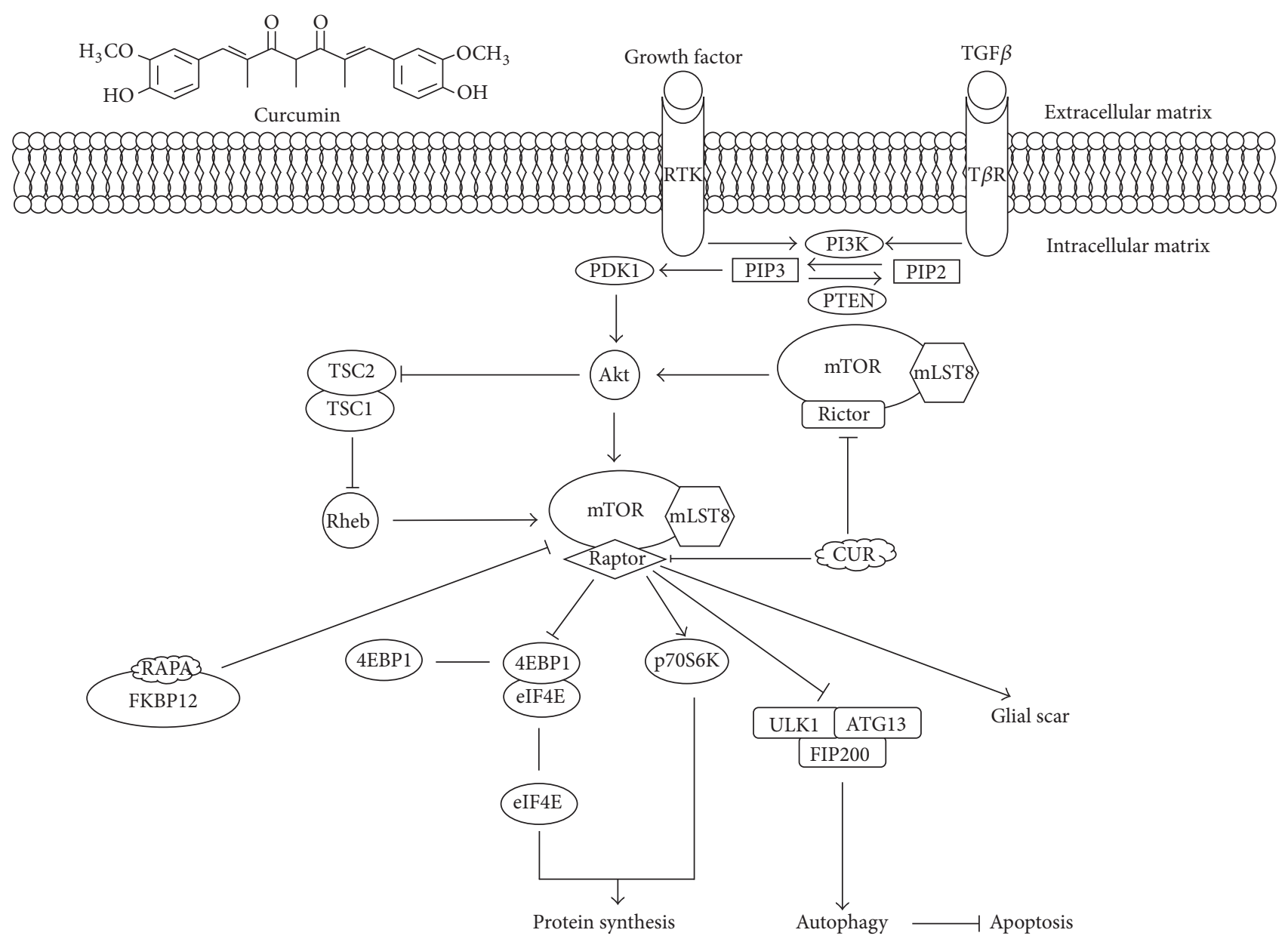

$\begin{array}{ll}\longrightarrow \text { Stimulation } & \text { CUR: curcumin } \\ \text { Inhibition } & \text { RAPA: rapamycin }\end{array}$

FIGURE 2: The mTOR signaling pathway and the possibility of curcumin's pharmacological action on the pathway in the treatment of SCI.

and vimentin, as well as manipulating CSPGs expression, also improving axon's regeneration into the lesion sites; those enhance the locomotor function after SCI [21]. Interestingly, the expression of CSPGs and the transforming growth factor$\beta$ (TGF- $\beta$ ) are upregulated after SCI through non-Smaddependent activation of the PI3K/Akt/mTOR signaling pathway, rather than the $S \operatorname{mad} 2 / 3$ signaling pathway [22], but the mechanism still remains unknown. Furthermore, epidermal growth factor receptor (EGR) is performing tyrosine kinase's activity with another term of receptor tyrosine kinase (RTK) within cell membrane, is upregulated in astrocytes after SCI and Ras homolog that are enriched in brain (Rheb), and is essential for mTOR activation through EGR; treatment with rapamycin reduces the expression of GFAP and vimentin, which inhibits the reactive gliosis [23] ( Figure 2).

3.2. Promoting Axon Regeneration Depends on Neuron Intrinsic Mechanism via Activating the Pathway after SCI. The constitutive expression activates Rheb in adult neurons after complete SCI enhances axon regeneration cross a ChABCtreated glial scar [24]. However, researches show that it does not eliminate the glial scar instead it made axon regeneration possible for the injured spinal cord. When neural stem cells are transplanted to specific sites in the injured spinal cord, it differentiates into multiple cellular phenotypes including neurons, which extend axons over striking distances and form ample synapses with resident cell [25]. Other researches mostly aim at the PTEN, for example, Liu et al. [26]. It has been reported that before complete spinal cord crush, conditional deletion of the PTEN gene promotes compensatory sprouting of uninjured corticospinal axons; it is also supposed that the regeneration of a large number of injured corticospinal tract (CST) axons crosses the injury site; besides, synapses in spinal segments distal to the injury were reformed by these regenerating CST axons, even though nothing was done to the glial scar formation. What is more, the codeletion of PTEN and suppressor of cytokine signaling 3 (SOCS3) could lead to the change of uninjured CST axons to denervation of the spinal cord [27]. Zukor et al. [28] show that suppression of PTEN's expression with shorthairpin RNA (shRNA) in the neonatal cortex also promotes the regeneration of injured CST axons and even promotes 
its functional recovery by combining with the treatment of Salmon Fibrin after SCI [29]. Following researches indicate that PTEN deletion shortly after SCI could enhance the regenerative growth of CST axons and motor function [30]. Moreover, the treatment of PTEN's inhibitor peroxovanadium and PTEN's antagonist peptides plays a similar role as PTEN's deletion in the functional recovery [31, 32]. Strikingly, PTEN's deletion promotes regrowth of CST axons even 1 year after chronic SCI [33]. The long-term consequences of conditional genetic deletion of PTEN in the sensorimotor cortex are the only cause of neuronal hypertrophy but no other detectable neuropathology [34]. Yet, there was an agedependent decline in axon regeneration with PTEN deletion. Compared with young mice, middle-aged to aging mice lose the effects in enhancing axon's regeneration distal to the injury [35]. In addition, mTOR signaling pathway activated by ATP, IL-6, and exercise as well as intermittent hypoxia training also improved the ability of translocating recovery after SCI, followed with the axon's regenerative potential of neurons [36-40].

3.3. Inducing Autophagy via Inhibiting the Pathway and Against the Apoptosis after SCI. Autophagy, called "selfeating" refers to an intracellular mechanism involved in the degradation of organelles and long-living proteins lysosomedependent pathway as well as for cleaning up dysfunctional cellular components; the kinase complex demands to stimulate autophagy in advance. ULK1/Atg13/FIP200 (unc-51like kinase 1/mammalian autophagy-related gene 13/focal adhesion kinase family-interacting protein about $200 \mathrm{kDa}$ ) are directly phosphorylated and suppressed by mTORC1 [15]. LC-3 and Beclin-1 are reliable markers of autophagy and improved autophagy. Rapamycin could decrease the phosphorylation of the p70 S6 kinase (p70S6K) protein and led to higher expression levels of LC-3 II and Beclin-1 in the spinal cord injury along with less TUNEL positive cell; it also acted against the apoptosis and reduced neural tissue damage with locomotor impairment after SCI [20,41]. Likewise, Simvastatin performed a similar role like Rapamycin in SCI by reducing mTOR protein and p70S6K phosphorylation, while Beclin-1 and LC-3 II are obviously enhanced [42]. Similarly, disturbed autophagy also plays a pivotal role in the pathogenesis of cardiomyopathy associated with uncorrected obesity named cardiomyopathy of obesity [43], and suppression of mTOR may act as a possible approach to hinder pathological cardiac hypertrophy by saving interrupted autophagic flux [44]. More surprisingly, without influencing phosphorylation of Akt, mTOR-independent autophagy inducer trehalose may ameliorate insulin resistance-induced myocardial contractile defect and apoptosis, by means of autophagy associated with dephosphorylation of $\mathrm{p} 38$ mitogen activated protein kinase (p38 MAPK) and Forkhead box O 1 (Foxol) [45]. On the contrary, several investigations have proved that the activation of Akt/mTOR signaling pathway inhibits the excessive autophagy and enhances the recovery following SCI $[46,47]$. Hence, only inducing autophagy appropriately could be beneficial to the recovery of SCI. Apoptosis is the process of programmed cell death and entails a series of characteristic cell changes, playing a crucial role in cell death and autophagy. Interestingly, there is an intimate link between apoptosis and autophagy in SCI, and the induction of autophagy could produce neuroprotective effects in acute spinal cord injury in rats via inhibition of apoptosis [48-51] (Figure 2).

\section{Curcumin Inhibits the mTOR Signaling Pathway}

Curcumin, a pleiotropic molecule from Curcuma longa, was first found to have antibacterial action in 1949 [52]. Extensive preclinical investigations over the past half century have indicated that curcumin and its analogs overcome the property of low bioavailability, fast metabolism, and bad chemical stability in the clinical application (e.g., C66, MC37, and EF24), which attracted considerable attention holding a gigantic potential in defending and curing several of inflammatory conditions and chronic diseases in cancer [53-55], diabetes and its associated complications [56, 57], diabetic cardiomyopathy [58-60] and diabetic nephropathy [61-63], cardiovascular diseases [64], CNS disorder/trauma [65], and others [66].

At present, curcumin, a well known anticancer medicine entering phase III clinical trials approved by FDA, aims at several signaling pathways and demonstrated the suppression of mTOR signaling pathway $[67,68]$. While the treatment of curcumin in SCI related to mTOR signaling pathway remains unreported, the pathway inhibited by curcumin in tumors has been reviewed [9]; 197 proteins were greatly recognized as curcumin binding target from HCT116 Colon Cancer Cell Line, and target is enriched in the nucleus, mitochondria, and plasma membrane; functional analyses show that the cellular protein synthesis downregulation of curcumin can induce autophagy and lysosomal activation [69]. In addition, discovery indicates that curcumin is a direct inhibitor of mTOR, which may remove the association of Raptor with mTOR at low concentration and suppress the binding of Rictor with mTOR at a high concentration [70], but it is not supported by molecular insight into the regulation. Therefore, a much more detailed mechanism about the inhibition of curcumin in mTOR signaling pathway should be further studied (Figure 2).

\section{Curcumin Inhibits Glial Scar Formation and Apoptosis after SCI}

Previous researches have already shown the positive influence of curcumin on the treatment of SCI, and a meta-analysis revealed the neurological recovery effect of curcumin [9]. The expression of GFAP and CSPG proteins is significantly suppressed by curcumin which inhibits the glial scar's formation both intracellularly and extracellularly and ameliorates the microenvironment for axon's regeneration [71-73]. CDGSH iron sulfur domain 2 (CISD2) was regarded as a survival gene like bcl-2 and also known to have role in antiapoptosis, which was showed to exert antiapoptotic effect in neural cells. Curcumin could attenuate the downregulation of CISD2 in SCI [74]. In addition, TUNEL (transferase-mediated dUTP nick end labeling) analysis in some investigations provided 
a great proof for antiapoptosis activity of curcumin in SCI treatment [75-77] (Figure 2).

\section{Conclusion}

The mTOR signaling pathway is involved in the axon regeneration and autophagy in the pathological process of SCI. While curcumin is also well known as a multiple targets medicine that can inhibit the mTOR signaling pathway in numerous diseases, likewise, the mentioned evidence above indicates that mTOR signaling pathway is a potential therapeutic target of curcumin in the treatment of SCI by ameliorating the microenvironment of axons regeneration which induced autophagy appropriately as well as having little impact on the intrinsic mechanism of the axon regeneration. However, there is still no investigations referring to the curcumin's influence involved in mTOR signaling pathway; thus another research should be done to make the related mechanisms about it clear, and it will provide another strong evidence for the potential utility of curcumin through the treatment of SCI.

$\begin{array}{ll}\text { Abbreviations } \\ \text { SCI: } & \text { Spinal cord injury } \\ \text { mTOR: } & \text { Mammalian target of rapamycin } \\ \text { TNF- } \alpha: & \text { Tumor necrosis factor- } \alpha \\ \text { IL: } & \text { Interleukin } \\ \text { PI3K: } & \text { Phosphoinositide 3-kinase } \\ \text { PIKK: } & \text { PI3K-related protein kinase } \\ \text { TBI: } & \text { Traumatic brain injury } \\ \text { GFAP: } & \text { Glial fibrillary acidic protein } \\ \text { CSPGs: } & \text { Chondroitin sulfate proteoglycans } \\ \text { ChABC: } & \text { Chondroitinase ABC } \\ \text { FKBP12: } & \text { The 12 kDa FK506-binding protein } \\ \text { PTEN: } & \text { Phosphatase and tensin homolog deleted } \\ & \text { on chromosome 10 } \\ \text { TGF- } \beta: & \text { Transforming growth factor- } \beta \\ \text { EGR: } & \text { Epidermal growth factor receptor } \\ \text { RTK: } & \text { Receptor tyrosine kinase } \\ \text { Rheb: } & \text { Ras homolog enriched in brain } \\ \text { CST: } & \text { Corticospinal tract } \\ \text { SOCS3: } & \text { Suppressor of cytokine signaling } 3 \\ \text { shRNA: } & \text { Short-hairpin RNA } \\ \text { p70S6K: } & \text { p70 S6 kinase } \\ \text { p38 MAPK: } & \text { p38 mitogen activated protein kinase } \\ \text { Foxol: } & \text { Forkhead box O 1 } \\ \text { CISD2: } & \text { CDGSH iron sulfur domain } 2 . \\ & \\ \end{array}$

\section{Conflicts of Interest}

The authors declare that they have no conflicts of interest.

\section{Authors' Contributions}

Xuehong Liu participated in this study design. Jingquan Lin prepared the first draft of the manuscript. Jingquan Lin, Xue
Huo, and Xuehong Liu participated in the revision of the manuscript and approved the final paper.

\section{Acknowledgments}

This work was supported by the Natural Science Foundation of Zhejiang Province (no. LY15H170001), the Public Technology Applied Research Projects Foundation of Shaoxing City (nos. 2013D10035 and 2015B70040), and the National Undergraduate Innovation Training Program (no. 201610349007).

\section{References}

[1] A. Singh, L. Tetreault, S. Kalsi-Ryan, A. Nouri, and M. G. Fehlings, "Global prevalence and incidence of traumatic spinal cord injury," Clinical Epidemiology, vol. 6, pp. 309-331, 2014.

[2] N. A. Silva, N. Sousa, R. L. Reis, and A. J. Salgado, "From basics to clinical: a comprehensive review on spinal cord injury," Progress in Neurobiology, vol. 114, pp. 25-57, 2014.

[3] M. Berry, Z. Ahmed, P. Morgan-Warren, D. Fulton, and A. Logan, "Prospects for mTOR-mediated functional repair after central nervous system trauma," Neurobiology of Disease, vol. 85, pp. 99-110, 2016.

[4] W. Young, "Spinal cord regeneration," Cell Transplantation, vol. 23, no. 4-5, pp. 573-611, 2014.

[5] H. Kanno, H. Ozawa, A. Sekiguchi et al., "The role of mTOR signaling pathway in spinal cord injury," Cell Cycle, vol. 11, no. 17, pp. 3175-3179, 2012.

[6] S. Mukhopadhyay, P. K. Panda, N. Sinha, D. N. Das, and S. K. Bhutia, "Autophagy and apoptosis: where do they meet?" Apoptosis, vol. 19, no. 4, pp. 555-566, 2014.

[7] H.-J. Wu, J.-L. Pu, P. R. Krafft, J.-M. Zhang, and S. Chen, “The Molecular Mechanisms Between Autophagy and Apoptosis: Potential Role in Central Nervous System Disorders," Cellular and Molecular Neurobiology, vol. 35, no. 1, pp. 85-99, 2014.

[8] O. Oral, Y. Akkoc, O. Bayraktar, and D. Gozuacik, "Physiological and pathological significance of the molecular cross-talk between autophagy and apoptosis," Histology and Histopathology, vol. 31, no. 5, pp. 479-498, 2016.

[9] M. Yao, L. Yang, J. Wang et al., "Neurological recovery and antioxidant effects of curcumin for spinal cord injury in the rat: a network meta-analysis and systematic review," Journal of Neurotrauma, vol. 32, no. 6, pp. 381-391, 2015.

[10] C. Cerella, A. Gaigneaux, M. Dicato, and M. Diederich, "Antagonistic role of natural compounds in mTOR-mediated metabolic reprogramming," Cancer Letters, vol. 356, no. 2, pp. 251-262, 2015.

[11] C. S. Ahuja, S. Nori, L. Tetreault et al., "Traumatic Spinal Cord Injury-Repair and Regeneration," Neurosurgery, vol. 80, pp. S9-S22, 2017.

[12] H.-X. Yuan and K.-L. Guan, "Structural insights of mTOR complex 1," Cell Research, vol. 26, no. 3, pp. 267-268, 2016.

[13] C. H. S. Aylett, E. Sauer, S. Imseng et al., "Architecture of human mTOR complex 1," Science, vol. 351, no. 6268, pp. 48-52, 2016.

[14] H. Yang, D. G. Rudge, J. D. Koos, B. Vaidialingam, H. J. Yang, and N. P. Pavletich, "MTOR kinase structure, mechanism and regulation,” Nature, vol. 497, no. 7448, pp. 217-223, 2013.

[15] M. Laplante and D. M. Sabatini, "MTOR signaling in growth control and disease," Cell, vol. 149, no. 2, pp. 274-293, 2012. 
[16] J. Jaworski and M. Sheng, "The growing role of mTOR in neuronal development and plasticity," Molecular Neurobiology, vol. 34, no. 3, pp. 205-219, 2006.

[17] K. Switon, K. Kotulska, A. Janusz-Kaminska et al., "Molecular neurobiology of mTOR," Neuroscience, vol. 341, pp. 112-153, 2017.

[18] E. J. Bradbury, L. D. F. Moon, R. J. Popat et al., "Chondroitinase ABC promotes functional recovery after spinal cord injury," Nature, vol. 416, no. 6881, pp. 636-640, 2002.

[19] B. Wang, Z. Xiao, B. Chen et al., "Nogo-66 promotes the differentiation of neural progenitors into astroglial lineage cells through mTOR-STAT3 pathway," PLoS ONE, vol. 3, no. 3, Article ID e1856, 2008.

[20] Y. Goldshmit, S. Kanner, M. Zacs et al., "Rapamycin increases neuronal survival, reduces inflammation and astrocyte proliferation after spinal cord injury," Molecular and Cellular Neuroscience, vol. 68, pp. 82-91, 2015.

[21] C.-H. Chen, C.-S. Sung, S.-Y. Huang et al., "The role of the PI3K/Akt/mTOR pathway in glial scar formation following spinal cord injury," Experimental Neurology, vol. 278, pp. 27-41, 2016.

[22] N. Jahan and S. S. Hannila, "Transforming growth factor $\beta$ induced expression of chondroitin sulfate proteoglycans is mediated through non-Smad signaling pathways," Experimental Neurology, vol. 263, pp. 372-384, 2015.

[23] S. Codeluppi, C. I. Svensson, M. P. Hefferan et al., "The RhebmTOR pathway is upregulated in reactive astrocytes of the injured spinal cord," Journal of Neuroscience, vol. 29, no. 4, pp. 1093-1104, 2009.

[24] D. Wu, M. C. Klaw, T. Connors, N. Kholodilov, R. E. Burke, and V. J. Tom, "Expressing constitutively active rheb in adult neurons after a complete spinal cord injury enhances axonal regeneration beyond a chondroitinase-treated glial scar," The Journal of Neuroscience, vol. 35, no. 31, pp. 11068-11080, 2015.

[25] P. Lu, Y. Wang, L. Graham et al., "Long-distance growth and connectivity of neural stem cells after severe spinal cord injury," Cell, vol. 150, no. 6, pp. 1264-1273, 2012.

[26] K. Liu, Y. Lu, J. K. Lee et al., "PTEN deletion enhances the regenerative ability of adult corticospinal neurons," Nature Neuroscience, vol. 13, no. 9, pp. 1075-1081, 2010.

[27] D. Jin, Y. Liu, F. Sun, X. Wang, X. Liu, and Z. He, "Restoration of skilled locomotion by sprouting corticospinal axons induced by co-deletion of PTEN and SOCS3," Nature Communications, vol. 6, article 8074, 2015.

[28] K. Zukor, S. Belin, C. Wang, N. Keelan, X. Wang, and Z. He, "Short hairpin RNA against PTEN enhances regenerative growth of corticospinal tract axons after spinal cord injury," The Journal of Neuroscience, vol. 33, no. 39, pp. 15350-15361, 2013.

[29] G. Lewandowski and O. Steward, "AAVshRNA-mediated suppression of PTEN in adult rats in combination with salmon fibrin administration enables regenerative growth of corticospinal axons and enhances recovery of voluntary motor function after cervical spinal cord injury," Journal of Neuroscience, vol. 34, no. 30, pp. 9951-9962, 2014.

[30] C. A. Danilov and O. Steward, "Conditional genetic deletion of PTEN after a spinal cord injury enhances regenerative growth of CST axons and motor function recovery in mice," Experimental Neurology, vol. 266, pp. 147-160, 2015.

[31] C. L. Walker and X.-M. Xu, "PTEN inhibitor bisperoxovanadium protects oligodendrocytes and myelin and prevents neuronal atrophy in adult rats following cervical hemicontusive spinal cord injury," Neuroscience Letters, vol. 573, pp. 64-68, 2014.

[32] Y. Ohtake, D. Park, P. M. Abdul-Muneer et al., "The effect of systemic PTEN antagonist peptides on axon growth and functional recovery after spinal cord injury," Biomaterials, vol. 35, no. 16, pp. 4610-4626, 2014.

[33] K. Du, S. Zheng, Q. Zhang et al., "Pten deletion promotes regrowth of corticospinal tract axons 1 year after spinal cord injury," Journal of Neuroscience, vol. 35, no. 26, pp. 9754-9763, 2015.

[34] E. A. Gutilla, M. M. Buyukozturk, and O. Steward, "Long-term consequences of conditional genetic deletion of PTEN in the sensorimotor cortex of neonatal mice," Experimental Neurology, vol. 279, pp. 27-39, 2016.

[35] C. G. Geoffroy, B. J. Hilton, W. Tetzlaff, and B. Zheng, "Evidence for an Age-Dependent Decline in Axon Regeneration in the Adult Mammalian Central Nervous System," Cell Reports, vol. 15, no. 2, pp. 238-246, 2016.

[36] L. Y. Hu, Z. G. Sun, Y. M. Wen et al., "ATP-mediated protein kinase B Akt/mammalian target of rapamycin mTOR/p70 ribosomal 66 protein $\mathrm{p} 70$ S6 kinase signaling pathway activation promotes improvement of locomotor function after spinal cord injury in rats," Neuroscience, vol. 169, no. 3, pp. 1046-1062, 2010.

[37] P. Yang, H. Wen, S. Ou, J. Cui, and D. Fan, "IL-6 promotes regeneration and functional recovery after cortical spinal tract injury by reactivating intrinsic growth program of neurons and enhancing synapse formation," Experimental Neurology, vol. 236, no. 1, pp. 19-27, 2012.

[38] P. Yang, Y. Qin, C. Bian, Y. Zhao, and W. Zhang, "Intrathecal delivery of IL-6 reactivates the intrinsic growth capacity of pyramidal cells in the sensorimotor cortex after spinal cord injury," PLoS ONE, vol. 10, no. 5, Article ID e0127772, 2015.

[39] G. Liu, M. R. Detloff, K. N. Miller, L. Santi, and J. D. Houlé, "Exercise modulates microRNAs that affect the PTEN/mTOR pathway in rats after spinal cord injury," Experimental Neurology, vol. 233, no. 1, pp. 447-456, 2012.

[40] D. V. Gutierrez, M. Clark, O. Nwanna, and W. J. Alilain, "Intermittent hypoxia training after $\mathrm{C} 2$ hemisection modifies the expression of PTEN and mTOR," Experimental Neurology, vol. 248, pp. 45-52, 2013.

[41] A. Sekiguchi, H. Kanno, H. Ozawa, S. Yamaya, and E. Itoi, "Rapamycin promotes autophagy and reduces neural tissue damage and locomotor impairment after spinal cord injury in mice," Journal of Neurotrauma, vol. 29, no. 5, pp. 946-956, 2012.

[42] K. Gao, G. Wang, Y. Wang et al., "Neuroprotective Effect of Simvastatin via Inducing the Autophagy on Spinal Cord Injury in the Rat Model," BioMed Research International, vol. 2015, Article ID 260161, 2015.

[43] Y. Zhang and J. Ren, "Epigenetics and obesity cardiomyopathy: From pathophysiology to prevention and management," Pharmacology and Therapeutics, vol. 161, pp. 52-66, 2016.

[44] Y. Zhang, X. Xu, and J. Ren, "MTOR overactivation and interrupted autophagy flux in obese hearts: A dicey assembly?" Autophagy, vol. 9, no. 6, pp. 939-941, 2013.

[45] Q. Wang and J. Ren, "mTOR-Independent autophagy inducer trehalose rescues against insulin resistance-induced myocardial contractile anomalies: Role of p38 MAPK and Foxol," Pharmacological Research, vol. 111, pp. 357-373, 2016.

[46] H.-Y. Zhang, Z.-G. Wang, F.-Z. Wu et al., "Regulation of autophagy and ubiquitinated protein accumulation by bFGF promotes functional recovery and neural protection in a rat 
model of spinal cord injury," Molecular Neurobiology, vol. 48, no. 3, pp. 452-464, 2013.

[47] C. L. Walker, M. J. Walker, N.-K. Liu et al., "Systemic bisperoxovanadium activates Akt/mTOR, reduces autophagy, and enhances recovery following cervical spinal cord injury," PLoS ONE, vol. 7, no. 1, Article ID e30012, 2012.

[48] Z.-Y. Wang, J.-H. Lin, A. Muharram, and W.-G. Liu, "Beclin1-mediated autophagy protects spinal cord neurons against mechanical injury-induced apoptosis," Apoptosis, vol. 19, no. 6, pp. 933-945, 2014.

[49] L. Zhang, P. Tang, H. Hou et al., "Autophagy reduces neuronal damage and promotes locomotor recovery via inhibition of apoptosis after spinal cord injury in rats," Molecular Neurobiology, vol. 49, no. 1, pp. 276-287, 2014.

[50] K.-L. Zhou, Y.-F. Zhou, K. Wu et al., "Stimulation of autophagy promotes functional recovery in diabetic rats with spinal cord injury," Scientific Reports, vol. 5, Article ID 17130, 2015.

[51] Y. Guo, S. Liu, X. Zhang et al., "G-CSF promotes autophagy and reduces neural tissue damage after spinal cord injury in mice," Laboratory Investigation, vol. 95, no. 12, pp. 1439-1449, 2015.

[52] E. Schraufstätter and H. Bernt, "Antibacterial action of curcumin and related compounds," Nature, vol. 164, no. 4167, pp. 456-457, 1949.

[53] P. A. Subramani, K. Panati, and V. R. Narala, "Curcumin Nanotechnologies and Its Anticancer Activity," Nutrition and Cancer, vol. 69, no. 3, pp. 381-393, 2017.

[54] B. Liang, Z. Liu, Y. Cao et al., "MC37, a new monocarbonyl curcumin analog, induces G2/M cell cycle arrest and mitochondria-mediated apoptosis in human colorectal cancer cells," European Journal of Pharmacology, vol. 796, pp. 139-148, 2017.

[55] S. Bisht, M. Schlesinger, A. Rupp et al., "A liposomal formulation of the synthetic curcumin analog EF24 (Lipo-EF24) inhibits pancreatic cancer progression: Towards future combination therapies," Journal of Nanobiotechnology, vol. 14, no. 1, article no. 57, 2016.

[56] A. S. Jiménez-Osorio, A. Monroy, and S. Alavez, "Curcumin and insulin resistance-Molecular targets and clinical evidences," BioFactors, vol. 42, no. 6, pp. 561-580, 2016.

[57] S. Rivera-Mancía, M. C. Lozada-García, and J. PedrazaChaverri, "Experimental evidence for curcumin and its analogs for management of diabetes mellitus and its associated complications," European Journal of Pharmacology, vol. 756, pp. 30-37, 2015.

[58] J. Ren and J. R. Sowers, "Application of a novel curcumin analog in the management of diabetic cardiomyopathy," Diabetes, vol. 63, no. 10, pp. 3166-3168, 2014.

[59] Y. Pan, Y. Wang, Y. Zhao et al., "Inhibition of JNK phosphorylation by a novel curcumin analog prevents high glucoseinduced inflammation and apoptosis in cardiomyocytes and the development of diabetic cardiomyopathy," Diabetes, vol. 63, no. 10, pp. 3497-3511, 2014.

[60] Y. Wang, S. Zhou, W. Sun et al., "Inhibition of JNK by novel curcumin analog C66 prevents diabetic cardiomyopathy with a preservation of cardiac metallothionein expression," American Journal of Physiology - Endocrinology and Metabolism, vol. 306, no. 11, pp. E1239-E1247, 2014.

[61] H. Wu, L. Kong, Y. Tan et al., "C66 ameliorates diabetic nephropathy in mice by both upregulating NRF2 function via increase in miR-200a and inhibiting miR-21," Diabetologia, vol. 59, no. 7, pp. 1558-1568, 2016.
[62] Y. Wang, Y. Wang, M. Luo et al., "Novel curcumin analog C66 prevents diabetic nephropathy via JNK pathway with the involvement of p300/CBP-mediated histone acetylation," Biochimica et Biophysica Acta-Molecular Basis of Disease, vol. 1852, no. 1, pp. 34-46, 2015.

[63] Y. Pan, Y. Huang, Z. Wang et al., "Inhibition of MAPK-mediated ACE expression by compound C66 prevents STZ-induced diabetic nephropathy," Journal of Cellular and Molecular Medicine, vol. 18, no. 2, pp. 231-241, 2014.

[64] S. Jiang, J. Han, T. Li et al., "Curcumin as a potential protective compound against cardiac diseases," Pharmacological Research, vol. 119, pp. 373-383, 2017.

[65] F. Ullah, A. Liang, A. Rangel et al., "High bioavailability curcumin: an anti-inflammatory and neurosupportive bioactive nutrient for neurodegenerative diseases characterized by chronic neuroinflammation," Archives of Toxicology, vol. 91, no. 4, pp. 1623-1634, 2017.

[66] S. C. Gupta, S. Patchva, and B. B. Aggarwal, "Therapeutic roles of curcumin: lessons learned from clinical trials," AAPS Journal, vol. 15, no. 1, pp. 195-218, 2013.

[67] M. Pulido-Moran, J. Moreno-Fernandez, C. Ramirez-Tortosa, and M. Ramirez-Tortosa, "Curcumin and health," Molecules, vol. 21, no. 3, article E264, 2016.

[68] G. Kumar, S. Mittal, K. Sak, and H. S. Tuli, "Molecular mechanisms underlying chemopreventive potential of curcumin: Current challenges and future perspectives," Life Sciences, vol. 148, pp. 313-328, 2016.

[69] J. Wang, J. Zhang, C.-J. Zhang et al., "In situ Proteomic Profiling of Curcumin Targets in HCT116 Colon Cancer Cell Line," Scientific Reports, vol. 6, Article ID 22146, 2016.

[70] C. S. Beevers, L. Chen, L. Liu, Y. Luo, N. J. G. Webster, and S. Huang, "Curcumin disrupts the mammalian target of rapamycin-raptor complex," Cancer Research, vol. 69, no. 3, pp. 1000-1008, 2009.

[71] J. Yuan, M. Zou, X. Xiang et al., "Curcumin improves neural function after spinal cord injury by the joint inhibition of the intracellular and extracellular components of glial scar," Journal of Surgical Research, vol. 195, no. 1, pp. 235-245, 2015.

[72] Y.-F. Wang, J.-N. Zu, J. Li, C. Chen, C.-Y. Xi, and J.-L. Yan, "Curcumin promotes the spinal cord repair via inhibition of glial scar formation and inflammation," Neuroscience Letters, vol. 560, pp. 51-56, 2014.

[73] L. M. Urdzikova, K. Karova, J. Ruzicka et al., "The antiinflammatory compound curcumin enhances locomotor and sensory recovery after spinal cord injury in rats by immunomodulation," International Journal of Molecular Sciences, vol. 17, no. 1, article no. 49, 2015.

[74] C.-C. Lin, T.-H. Chiang, W.-J. Chen, Y.-Y. Sun, Y.-H. Lee, and M.-S. Lin, "CISD2 serves a novel role as a suppressor of nitric oxide signalling and curcumin increases CISD2 expression in spinal cord injuries," Injury, vol. 46, no. 12, pp. 2341-2350, 2015.

[75] H. Ni, W. Jin, T. Zhu et al., "Curcumin modulates TLR4/NF$\kappa \mathrm{B}$ inflammatory signaling pathway following traumatic spinal cord injury in rats," Journal of Spinal Cord Medicine, vol. 38, no. 2, pp. 199-206, 2015.

[76] H. Ni, W. Jin, B. Yuan et al., "Curcumin inhibits the increase of labile zinc and the expression of inflammatory cytokines after traumatic spinal cord injury in rats," Journal of Surgical Research, vol. 187, no. 2, pp. 646-652, 2014.

[77] W. Jin, J. Wang, T. Zhu et al., "Anti-inflammatory effects of curcumin in experimental spinal cord injury in rats," Inflammation Research, vol. 63, no. 5, pp. 381-387, 2014. 

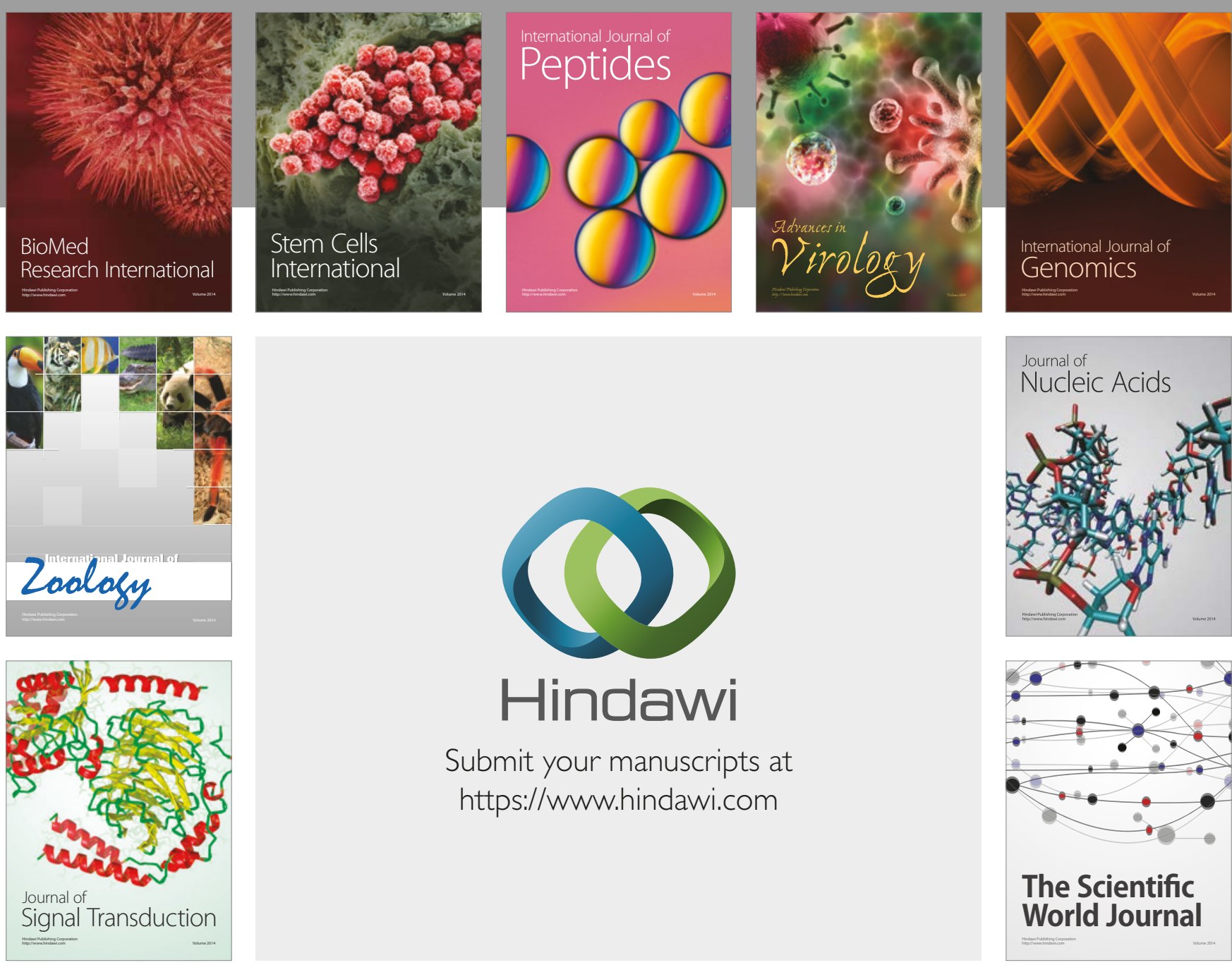

Submit your manuscripts at

https://www.hindawi.com
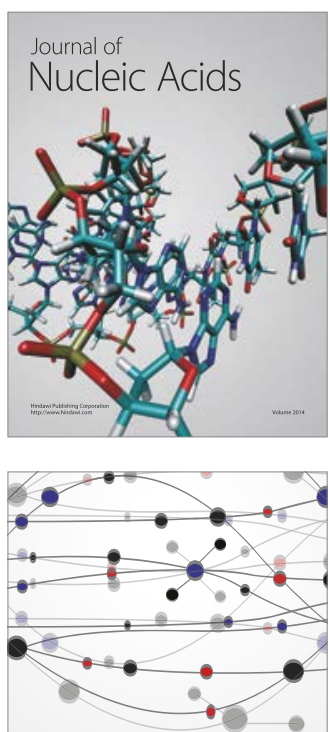

The Scientific World Journal

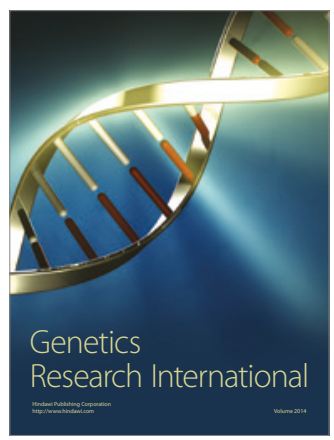

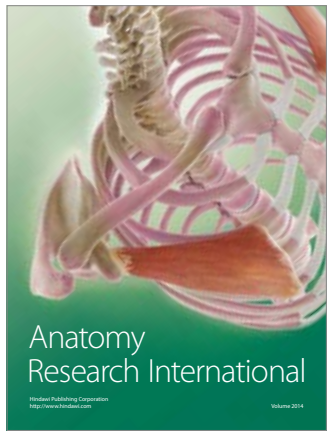

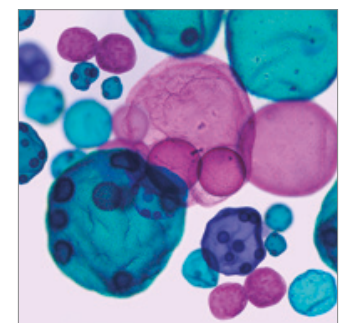

International Journal of Microbiology
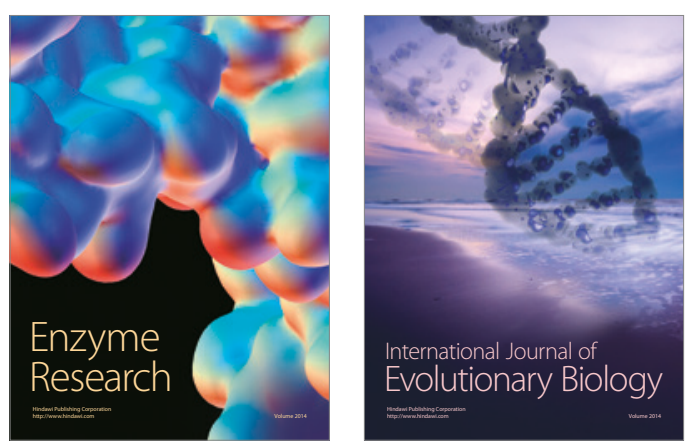
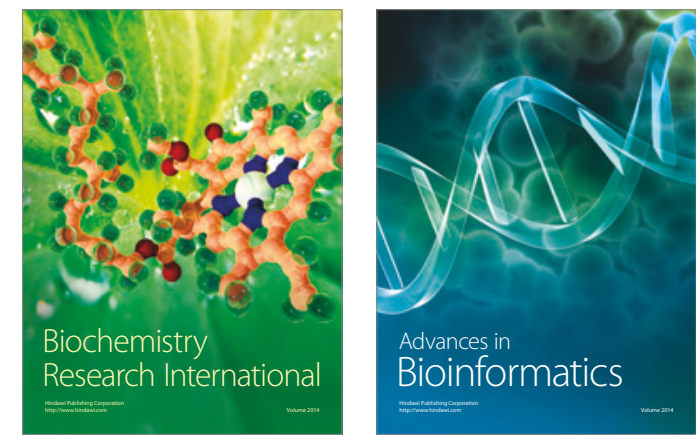

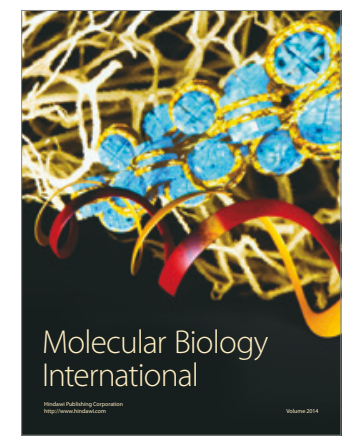

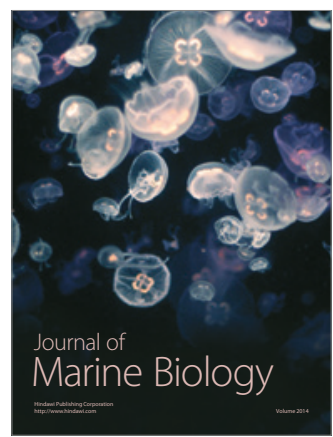

